\title{
Isospectral Hamiltonian for position-dependent mass for an arbitrary quantum system and coherent states
}

\author{
Sid-Ahmed Yahiaoui and Mustapha Bentaiba* \\ LPTHIRM, département de physique, \\ faculté des sciences, université Sâ̂d DAHLAB-Blida1, \\ B.P. 270 Route de Soumâa, 09000 Blida, Algeria
}

(Date textdate; Received textdate; Revised textdate; Accepted textdate; Published textdate)

\begin{abstract}
By means of the unitary transformation, a new way for discussing the ordering prescription of Schrödinger equation with a position-dependent mass (PDM) for isospectral Hamiltonian operators is presented. We show that the ambiguity parameter choices in the kinetic part of the Hamiltonian can be explained through an exact SUSY symmetry as well as a consequence of an accidental symmetry under the $\mathbb{Z}_{2}$ action. By making use of the unitary transformation, we construct coherent states for a family of PDM isospectral Hamiltonians from a suitable choice of ladder operators. We show that these states preserve the usual structure of Klauder-Perelomov's states and thus saturate and minimize the generalized position-momentum uncertainty relation (GUR) under some special restrictions. We show that GUR's properties can be used to determine the sign of the superpotential.

PACS numbers: 03.65.Fd, 02.30.+b
\end{abstract}

Keywords: Isospectral Hamiltonian, Unitary transformation, Position-dependent mass, Coherent states

*Electronic address: bentaiba@univ-blida.dz

Typeset by REVTEX 


\section{INTRODUCTION}

Nowadays, it is well-known that a family of Hamiltonians with the same eigenvalues set of the original Hamiltonian is called isospectral Hamiltonians [1, 2]. These families have been known for a long time and were obtained by means of factorization method [3], the Gel'fand-Levitan's approach [4], supersymmetric quantum mechanics (SUSY) and the related shape-invariant [5] and Lie algebraic procedure [6]. These techniques are known as a most fruitful approaches for solving Schrödinger equation in the context of constant mass.

When working with quantum system subjected to interact with a given interaction, however, usually considerations impose to identify the mass-term with the concept of effective mass. By a way, such quantum system becomes position-dependent mass (PDM) and considerable interest has been recently devoted in studying the Schrödinger equation under this new perspective [7-17]. We find the most extensive use of such an equation in effective interactions in nuclear physics [18], in the framework of curved spaces [19], in the point of view of $\mathcal{P} \mathcal{T}$-symmetry [20, 21], quantum wells, wires and dots [22] and semiconductor heterostructures [23]. May be the important concept in the framework of PDM concerns the problem of choosing some fixed ordering prescription in the kinetic part of the Hamiltonian, coming from noncommutativity between the mass-term $m(x)$ and momentum $\widehat{P}$ operator.

This problem is a long standing one in quantum mechanics and up to now some uncertainties are concerned about the form of the kinetic energy term $\widehat{T}$ in the Hamiltonian. To cope with this difficulty, it was stressed by von Roos [24] that the correct kinetic part of the Hamiltonian can be written as

$$
h_{\mathrm{vR}}=\frac{1}{4}\left(M^{a^{\prime}}(x) \widehat{P} M^{b^{\prime}}(x) \widehat{P} M^{c^{\prime}}(x)+M^{c^{\prime}}(x) \widehat{P} M^{b^{\prime}}(x) \widehat{P} M^{a^{\prime}}(x)\right)+V(x),
$$

which has an advantage to keep $h_{\mathrm{vR}}$ hermitian. Here $V(x)$ is a potential and $\widehat{P}\left(\equiv-i \hbar \frac{d}{d x}\right)$ is the conventional momentum operator. $M(x)=m_{0} m(x)$ is the position-dependent mass function $\left(m(x)\right.$ being dimensionless and $m_{0}$ is a constant mass), and $a^{\prime}, b^{\prime}$ and $c^{\prime}$ are arbitrary parameters satisfying the condition: $a^{\prime}+b^{\prime}+c^{\prime}=-1$.

In the other context, a set of coherent states (CS) may be the most important set of basis vectors knows in mathematical physics and which are not necessarily orthonormals. Originally, they were first introduced for the harmonic oscillator by Schrödinger in 1926 [25], and latter by Glauber [26] who showed that these states describe the electromagnetic 
correlation functions in the quantum optics. These states are also constructed for a family of isospectral oscillator Hamiltonians in [27]. The construction of CS is approached by three different ways and lead to the equivalent state vector for harmonic oscillator (see, e.g., [28] and references therein). For instance, we can recall their definition: (i) as eigenstates of an annihilation operator (Barut-Girardello's approach), (ii) as a displaced version of the groundstate wave-function (Klauder-Perelomov's approach) and (iii) as minimum uncertainty states (Schrödinger's approach). Very recently, CS have also been constructed for a wide family of Hamiltonians endowed with PDM [29 32].

In this paper, our primary concern is to extend the procedure worked out in [33] to PDM endowed with an effective potential and construct their PDM CS. We want to tackle the problem of ordering ambiguity in a new way and differently than [31], and prove that there is a special ordering in PDM framework due essentially to the existence of the unitary transformation. Once the suitable factorization operators, $\mathcal{Q}_{\alpha}$ and $\mathcal{Q}_{\alpha}^{\dagger}$, have been chosen to work as ladders, we prove that selecting the appropriate SUSY-parameter values $\alpha$ can be interpreted as a consequence of both exact SUSY and accidental symmetries. We show that the last symmetry is implemented under the $\mathbb{Z}_{2}$-transformation, through an operator $\widehat{\mathcal{Z}}$, if and only if $\alpha=1 / 2$ is considered as the unique fixed point of the $\mathbb{Z}_{2}$ action. The related PDM CS are then constructed as a displaced version of the ground-state wave-function and minimize the generalized position-momentum uncertainty relation (GUR). The properties of GUR lead us to remark two important observations: (i) if $\alpha=1 / 2$ then the deduced PDM CS saturate completely GUR and (ii) PDM CS are minimized when $\alpha \neq 1 / 2$. We observe that the minimization allow us to fix the sign of the superpotential $\mathcal{W}_{\alpha}(x)$ appearing in both ladder operators.

The paper is organized as follows. We start by introducing in section 2 the family of PDM isospectral Hamiltonians, their eigenstates and a pair of associated ladder operators through the use of unitary transformation. In section 3 we show that there is a special ordering, that may be checked by a one SUSY parameter, to select the kinetic part of the Hamiltonian. The related PDM CS are constructed in section 4. They are shown to preserve the structure of Klauder-Perelomov's states, and saturate and minimize GUR under some special restrictions. Our concluding remarks are summarized in the last section. 


\section{EFFECTIVE MASS LADDER OPERATORS AND UNITARY TRANSFOR- MATION}

Here we follow Morrow and Brownstein [34] who have shown from (1.1) the constraint $a^{\prime}=$ $c^{\prime}$, by comparing exact solutions of some models with experimental results. The restricted Hamiltonian (1.1), with a new change in parameters $a^{\prime}=a$ and $b^{\prime}=2 b$, can be now written as (see, [31] )

$$
h_{a}=\frac{1}{2}\left(m^{a}(x) \widehat{P} m^{2 b}(x) \widehat{P} m^{a}(x)\right)+V(x),
$$

where we used the atomic units $\hbar=m_{0}=1$ and $2 a+2 b=-1$. Let us factorize (2.1) in terms of the operators

$$
\begin{aligned}
q_{a} & =\frac{1}{\sqrt{2}}\left(m^{a}(x) \frac{d}{d x} m^{b}(x)+w(x)\right), \\
q_{a}^{\dagger} & =\frac{1}{\sqrt{2}}\left(-m^{b}(x) \frac{d}{d x} m^{a}(x)+w(x)\right),
\end{aligned}
$$

where $w(x)$ is an arbitrary real-function. The Hamiltonians $q_{a}^{\dagger} q_{a}$ and $q_{a} q_{a}^{\dagger}$ are SUSY partners. It is well known that $q_{a}$ and $q_{a}^{\dagger}$ are not unique when the only requirement for $h_{a}$ is its hermiticity, then one can construct a family of strictly isospectral Hamiltonians through a new operators [33]

$$
\begin{aligned}
& Q_{a}=\frac{1}{\sqrt{2}}\left(m^{a}(x) \frac{d}{d x} m^{b}(x)+W(x)\right), \\
& Q_{a}^{\dagger}=\frac{1}{\sqrt{2}}\left(-m^{b}(x) \frac{d}{d x} m^{a}(x)+W(x)\right),
\end{aligned}
$$

such that

$$
Q_{a} Q_{a}^{\dagger}=q_{a} q_{a}^{\dagger}
$$

On setting $m(x)=U^{-2}(x)$, where $U(x)$ is some positive-definite function and substituting (2.2) and (2.3) into (2.4), one gets Riccati equation which can be solved to give:

$$
W(x)=w(x)+\phi_{\lambda}(x), \quad \text { with } \quad \phi_{\lambda}(x)=\frac{U(x)}{\lambda+\int^{x} \xi_{0}^{2}(\eta) d \eta} \xi_{0}^{2}(x),
$$

where $\lambda$ is a constant of integration and $\xi_{0}(x)$ is the normalized ground-state eigenfunction of the Hamiltonian $q_{a}^{\dagger} q_{a}$, given by

$$
\xi_{0}(x) \sim U^{-1-2 a}(x) \exp \left\{-\int^{\mu(x)} w(\eta) d \mu(\eta)\right\},
$$


up to normalization constant and for convenience we introduce the auxiliary mass function $\mu(x)=\int^{x} d \eta / U(\eta)$.

One can construct the eigenfunctions $\left|\Xi_{n}\right\rangle$ of the strictly isospectral family of $H_{a}=$ $Q_{a}^{\dagger} Q_{a}+\epsilon,\left(\epsilon\right.$ corresponds to the ground-state energy, i.e., $\left.\epsilon=E_{0}\right)$, which is intertwined with $h_{a}=q_{a}^{\dagger} q_{a}+\epsilon$ by means of the intertwining operator $\mathcal{B}_{a}=Q_{a}^{\dagger} q_{a}[13$, , 33]

$$
H_{a} \mathcal{B}_{a}=\mathcal{B}_{a} h_{a} \quad \Longrightarrow \quad\left(Q_{a}^{\dagger} Q_{a}\right) Q_{a}^{\dagger} q_{a}=Q_{a}^{\dagger} q_{a}\left(q_{a}^{\dagger} q_{a}\right)
$$

It turns out that the normalized eigenfunctions $\left|\Xi_{n}\right\rangle$ of $H_{a}$ can be written in terms of the eigenfunctions $\left|\xi_{n}\right\rangle$ as

$$
\left|\Xi_{n}\right\rangle=\frac{1}{E_{n}-\epsilon} Q_{a}^{\dagger} q_{a}\left|\xi_{n}\right\rangle, \quad(n=1,2,3, \cdots)
$$

It is worth noting from (2.7) that $\epsilon=E_{0} \notin \operatorname{Spect}\left(H_{a}\right)$. From (2.7), it is easy to determine the normalized eigenfunctions $\Xi_{n}(x)\left(\equiv\left\langle x \mid \Xi_{n}\right\rangle\right)$ in the coordinate representation and are given by

$$
\begin{aligned}
& \Xi_{0}(x) \sim \frac{\xi_{0}(x)}{\lambda+\int^{x} \xi_{0}^{2}(\eta) d \eta} \\
& \Xi_{n}(x)=\left(1+\frac{1}{2\left(E_{n}-\epsilon\right)} \phi_{\lambda}(x) q_{a}\right) \xi_{n}(x) .
\end{aligned}
$$

Thus the operators $Q_{a}$ and $q_{a}$ connect the states $\left|\xi_{n}\right\rangle$ and $\left|\Xi_{n}\right\rangle$ and can not be considered as creation and annihilation operators. To cope with this difficulty, we are interested now in identifying these set of ladder operators by introducing the unitary transformation, $\widehat{\mathfrak{U}}$, following Kumar and Khare [33]

$$
Q_{a}=q_{a} \widehat{\mathfrak{U}}^{\dagger}, \quad \text { and } \quad Q_{a}^{\dagger}=\widehat{\mathfrak{U}} q_{a}^{\dagger},
$$

where (2.4) implies that $\widehat{\mathfrak{U}}^{\dagger} \widehat{\mathfrak{U}} \equiv \widehat{\mathfrak{U}}^{\dagger}=\mathbb{1}$. Once more, let us consider the factorization

$$
\mathcal{H}_{a}=\mathcal{Q}_{a}^{\dagger} \mathcal{Q}_{a}+\epsilon
$$

by defining the new ladder operators $\mathcal{Q}_{a}$ and $\mathcal{Q}_{a}^{\dagger}$ in terms of $\widehat{\mathfrak{U}}$ and $\widehat{\mathfrak{U}}^{\dagger}$ as

$$
\mathcal{Q}_{a}=\widehat{\mathfrak{U}} q_{a} \widehat{\mathfrak{U}}^{\dagger} \equiv \widehat{\mathfrak{U}} Q_{a}, \quad \text { and } \quad \mathcal{Q}_{a}^{\dagger}=\widehat{\mathfrak{U}} q_{a}^{\dagger} \widehat{\mathfrak{U}}^{\dagger} \equiv Q_{a}^{\dagger} \widehat{\mathfrak{U}}^{\dagger},
$$

so that $Q_{a}^{\dagger} Q_{a}=\mathcal{Q}_{a}^{\dagger} \mathcal{Q}_{a}:=\widehat{\mathfrak{U}}\left(q_{a}^{\dagger} q_{a}\right) \widehat{\mathfrak{U}}^{\dagger}$ and leads to the relation $\mathcal{H}_{a}=\widehat{\mathfrak{U}} h_{a} \widehat{\mathfrak{U}}^{\dagger}$. A consequence of isospectrality between $h_{a}$ and $H_{a}$ implies that $E_{n} \equiv\left\langle\Xi_{n}\left|H_{a}\right| \Xi_{n}\right\rangle=\left\langle\xi_{n}\left|h_{a}\right| \xi_{n}\right\rangle$. 
Using (2.12) we get a relation connecting the basis $\left\{\left|\xi_{n}\right\rangle\right\}_{n \in \mathbb{N}}$ and $\left\{\left|\Xi_{n}\right\rangle\right\}_{n \in \mathbb{N}}$ as follows

$$
\left|\xi_{n}\right\rangle=\widehat{\mathfrak{U}}^{\dagger}\left|\Xi_{n}\right\rangle, \quad \text { and } \quad\left|\Xi_{n}\right\rangle=\widehat{\mathfrak{U}}\left|\xi_{n}\right\rangle
$$

Moreover, the relevance of relationships (2.10), (2.12) and (2.13) is clear by noting that

$$
\begin{aligned}
\left\langle\xi_{n}\left|q_{a}^{\dagger} q_{a}\right| \xi_{n}\right\rangle & =\left\langle\xi_{n}\left|\widehat{\mathfrak{U}}^{\dagger}\left(\widehat{\mathfrak{U}} q_{a}^{\dagger}\right)\left(q_{a} \widehat{\mathfrak{U}}^{\dagger}\right) \widehat{\mathfrak{U}}\right| \xi_{n}\right\rangle \\
& =\left\langle\widehat{\mathfrak{U}} \xi_{n}\left|Q_{a}^{\dagger} Q_{a}\right| \widehat{\mathfrak{U}} \xi_{n}\right\rangle \\
& =\left\langle Q_{a} \widehat{\mathfrak{U}} \xi_{n} \mid Q_{a} \widehat{\mathfrak{U}} \xi_{n}\right\rangle \equiv E_{n}-\epsilon,
\end{aligned}
$$

which leads to define a new state $\left|\Upsilon_{n}\right\rangle \propto Q_{a}\left(\widehat{\mathfrak{U}}\left|\xi_{n}\right\rangle\right)=Q_{a}\left|\Xi_{n}\right\rangle$, such that $\left\langle\Upsilon_{n} \mid \Upsilon_{n}\right\rangle=$ $\|\left|\Upsilon_{n}\right\rangle \|^{2}=E_{n}-\epsilon<\infty$. Then, the new set of states

$$
S_{n}=\left\{\left|\Upsilon_{n}\right\rangle=\frac{1}{\sqrt{E_{n}-\epsilon}} Q_{a}\left(\widehat{\mathfrak{U}}\left|\xi_{n}\right\rangle\right)=\frac{1}{\sqrt{E_{n}-\epsilon}} Q_{a}\left|\Xi_{n}\right\rangle \mid E_{n} \neq \epsilon\right\},
$$

consists of normalized eigenfunctions of $H_{a} \equiv \mathcal{H}_{a}=\mathcal{Q}_{a}^{\dagger} \mathcal{Q}_{a}+\epsilon$ belonging to the eigenvalues $E_{n}=\operatorname{Spect}\left(H_{a}\right)$. Now, following [31], let $\left|\Upsilon_{n, \epsilon}\right\rangle$ be a function which is orthogonal to the set $\left\{\left|\Upsilon_{n}\right\rangle\right\}_{n \in \mathbb{N}}$; i.e., $\left\langle\Upsilon_{n} \mid \Upsilon_{n, \epsilon}\right\rangle \equiv\left\langle Q_{a} \Xi_{n} \mid \Upsilon_{n, \epsilon}\right\rangle=\left\langle\Xi_{n} \mid Q_{a}^{\dagger} \Upsilon_{n, \epsilon}\right\rangle=0$. Then using (2.10), it is easy to convince ourselves that $Q_{a}^{\dagger}\left|\Upsilon_{n, \epsilon}\right\rangle \equiv \widehat{\mathfrak{U}}\left(q_{a}^{\dagger}\left|\Upsilon_{n, \epsilon}\right\rangle\right)=0$, since the state $\left|\Xi_{n}\right\rangle \neq 0$ and the related solution reads, in the coordinate representation, as

$$
\Upsilon_{n, \epsilon}(x) \sim \frac{1}{m^{a}(x)} \exp \left\{\int^{\mu(x)} w(\eta) d \mu(\eta)\right\} .
$$

If $\left\langle\Upsilon_{n, \epsilon} \mid \Upsilon_{n, \epsilon}\right\rangle<\infty$, then $\operatorname{Spect}\left(H_{a}\right)=\operatorname{Spect}\left(h_{a}\right) \cup\{\epsilon\}$ which leads to impose that $m(x)$ has no zeros and $a<0$ (i.e., $b>-1 / 2$ ). This problem is closely related to the ordering problem and we will face to this question in the next section.

In summary, $h_{a}$ and $H_{a}$ are isospectral operators and are related by the unitary transformation $\widehat{\mathfrak{U}}$. We would like also to emphasize that $\mathcal{Q}_{a}$ and $\mathcal{Q}_{a}^{\dagger}$, as they are defined in (2.12), are nothing but the correct set of creation and annihilation operators for our isospectral Hamiltonians.

\section{SUSY PARAMETER $\alpha$ AND ACCIDENTAL SYMMETRY $\mathbb{Z}_{2}$}

In this section we take full advantage of ladder operators derived under unitary transformation in the previous section. To this end, inserting (2.2a) into (2.12), the operator $\mathcal{Q}_{a}$ 
can be expressed as

$$
\mathcal{Q}_{a}=\widehat{\mathfrak{U}} q_{a} \widehat{\mathfrak{U}}^{\dagger} \equiv \frac{1}{\sqrt{2}}\left(\widehat{\mathfrak{U}} m^{a}(x) \frac{d}{d x} m^{b}(x) \widehat{\mathfrak{U}}^{\dagger}+\widehat{\mathfrak{U}} w(x) \widehat{\mathfrak{U}}^{\dagger}\right)
$$

By substituting $\widehat{\mathfrak{U}}^{\dagger} \widehat{\mathfrak{U}}=\mathbb{1}$ twice into the first term of the right-hand side of (3.1), taking into account that $b=-(1 / 2+a)$, the operators $\mathcal{Q}_{a}$ and $\mathcal{Q}_{a}^{\dagger}$ can be rewritten explicitly in the form:

$$
\begin{aligned}
\mathcal{Q}_{a} \longrightarrow \mathcal{Q}_{\alpha} & =\frac{1}{\sqrt{2}}\left(f^{\alpha}(x) \frac{d}{d x} g(x) f^{-\alpha}(x)+\mathcal{W}(x)\right), \\
\mathcal{Q}_{a}^{\dagger} \longrightarrow \mathcal{Q}_{\alpha}^{\dagger} & =\frac{1}{\sqrt{2}}\left(-g(x) f^{-\alpha}(x) \frac{d}{d x} f^{\alpha}(x)+\mathcal{W}(x)\right),
\end{aligned}
$$

where $f(x), g(x)$ and the superpotential $\mathcal{W}(x)$ are real functions defined following

$$
f^{\alpha}(x)=\widehat{\mathfrak{U}} f^{a}(x) \widehat{\mathfrak{U}}^{\dagger}, \quad g(x)=\widehat{\mathfrak{U}} m^{-1 / 2}(x) \widehat{\mathfrak{U}}^{\dagger}, \quad \text { and } \quad \mathcal{W}(x)=\widehat{\mathfrak{U}} w(x) \widehat{\mathfrak{U}}^{\dagger},
$$

where we assume that the new parameter $\alpha$ only labels a particular ordering in the kinetic part of the Hamiltonian. Then from (2.11), $\mathcal{H}_{a} \rightarrow \mathcal{H}_{\alpha}=\mathcal{Q}_{\alpha}^{\dagger} \mathcal{Q}_{\alpha}+\epsilon$, we have

$$
\mathcal{H}_{\alpha}=-\frac{1}{2} \frac{d}{d x} g^{2}(x) \frac{d}{d x}+\mathcal{V}_{\mathrm{eff}}^{(\alpha)}(x)+\epsilon,
$$

where $\mathcal{V}_{\text {eff }}^{(\alpha)}(x)$ is known as the effective potential. In order to relate (3.5) to PDM problem, we look for the functions $f(x)$ and $g(x)$ that satisfy the condition $f(x)=g(x)=U(x)$. Then (3.2) and (3.3) become

$$
\begin{aligned}
& \mathcal{Q}_{\alpha} \equiv \frac{1}{\sqrt{2}}\left(U^{\alpha}(x) \frac{d}{d x} U^{1-\alpha}(x)+\mathcal{W}_{\alpha}(x)\right), \\
& \mathcal{Q}_{\alpha}^{\dagger} \equiv \frac{1}{\sqrt{2}}\left(-U^{1-\alpha}(x) \frac{d}{d x} U^{\alpha}(x)+\mathcal{W}_{\alpha}(x)\right),
\end{aligned}
$$

where the effective potential, $\mathcal{V}_{\text {eff }}^{(\alpha)}(x)=\mathcal{V}_{\alpha}(x)+\mathcal{V}_{\mathrm{U}}^{(\alpha)}(x)$, is defined in terms of the superpotential $\mathcal{W}_{\alpha}(x)$ as

$$
\begin{aligned}
\mathcal{V}_{\alpha}(x)-\epsilon & =\frac{1}{2} \mathcal{W}_{\alpha}^{2}(x)-\frac{1}{2} U(x) \mathcal{W}_{\alpha}^{\prime}(x)+\frac{1-2 \alpha}{2} U^{\prime}(x) \mathcal{W}_{\alpha}(x), \\
\mathcal{V}_{\mathrm{U}}^{(\alpha)}(x) & =\frac{\alpha(\alpha-1)}{2} U^{\prime 2}(x)+\frac{\alpha-1}{2} U^{\prime \prime}(x) U(x)
\end{aligned}
$$

However, we have observed that the appropriate mapping: $\alpha \mapsto \alpha_{n}=n \alpha$, for $n \in \mathbb{N}-\{0\}$, has a remarkable property; it keeps the kinetic operator in (3.5) unchanged and we will see hereafter why these maps were chosen. We shall take full advantage of this last mapping, 
since the problem we have to deal with concerns the choice of the appropriate profile for mass functions $U(x)$, (i.e., $m(x))$, appearing in (3.6) and represented by the terms $\zeta^{(+)}=U^{\alpha_{n}}(x)$ and $\zeta^{(-)}=U^{1-\alpha_{n}}(x)$. Taking into account that $U(x)=m^{-1 / 2}(x)$, we are faced the situation in which two distinguishable profiles for $U(x)$ must be considered:

(P1) $\zeta^{( \pm)}(x)$ admit singularities (i.e., $m(x)$ has zeros). This implies that both exponents $(1-n \alpha, n \alpha)<0$, with $\alpha$ is confined to the domain: $\operatorname{dom}(\alpha)=(-\infty, 0] \cup\left[\frac{1}{n},+\infty\right)$. Then it is easy to realize that this case must be omitted in order to avoid a possible divergence of $\mathcal{Q}_{\alpha}$ (i.e., $\mathcal{H}_{\alpha}$ ), when $\alpha= \pm \infty$.

(P2) $\zeta^{( \pm)}(x)$ admit zeros (i.e., $m(x)$ has singularities). This implies that $(1-n \alpha, n \alpha)>0$ and a similar reasoning shows that $0 \leq \alpha \leq \frac{1}{n} \leq 1$, for $n=1,2,3, \cdots$.

The case (P2) is which we are looking for. Then it is evident that the functions $U(x)$ (resp. $m(x))$ are chosen in such a way that they accept zeros (resp. singularities). This fact suggest that the ordering in the kinetic operator depends closely on the choice of SUSY parameter $\alpha$ which belongs to the discrete and bounded set:

$$
\mathfrak{S}_{\alpha_{n}}=\left\{\forall n \in \mathbb{N}-\{0\} \mid \alpha_{\infty}=0, \quad \text { and } \quad \alpha_{n}=\frac{1}{n} \in \mathbb{Q}^{+}\right\} \equiv\left\{1, \frac{1}{2}, \frac{1}{3}, \ldots, \frac{1}{n}, \ldots, 0\right\},
$$

which is well-known that it is closed in $\mathbb{R}$, and hence compact. For instances, among the SUSY parameter $\alpha_{n}$ of the set (3.8), some of frequently used form of the kinetic operators are reported below in the table. In the ongoing analysis, we will suppress the $n$-index of all the parameters $\alpha$.

TABLE I: Some of mostly used forms of the kinetic operators for describing PDM system. Among those ambiguity parameter choices, we may quote those of: (i) Zhu and Kroemer (ZK) and (ii) BenDaniel and Duke (BDD) both used in describing the motion of electrons in compositionally graded crystals, and (iii) Bagchi, Banerjee, Quesne and Tkachuk (BBQT).

\begin{tabular}{|c|c|c|c|c|}
\hline \multirow{2}{*}{$\begin{array}{l}\text { Parameters } \\
(\alpha, n) \quad(a, b)\end{array}$} & \multicolumn{2}{|c|}{ Operators } & \multirow[b]{2}{*}{ (Туре) } & \multirow[b]{2}{*}{ Refs. } \\
\hline & $\mathcal{Q}_{\alpha}$ & $\widehat{T}_{\alpha}$ & & \\
\hline$(0, \infty)\left(-\frac{1}{2}, 0\right)$ & $\frac{1}{\sqrt{2}}\left(\frac{d}{d x} U(x)+\mathcal{W}_{0}(x)\right)$ & $\frac{1}{2} U(x) \widehat{P}^{2} U(x)$ & $(\mathrm{ZK})$ & [35] \\
\hline$(1,1) \quad\left(0,-\frac{1}{2}\right)$ & $\frac{1}{\sqrt{2}}\left(U(x) \frac{d}{d x}+\mathcal{W}_{1}(x)\right)$ & $\frac{1}{2} \widehat{P} U^{2}(x) \widehat{P}$ & $(\mathrm{BDD})$ & {$[36]$} \\
\hline$\left(\frac{1}{2}, 2\right) \quad\left(-\frac{1}{4},-\frac{1}{4}\right)$ & $\frac{1}{\sqrt{2}}\left(\sqrt{U(x)} \frac{d}{d x} \sqrt{U(x)}+\mathcal{W}_{1 / 2}(x)\right.$ & $\frac{1}{2} \sqrt{U(x)} \widehat{P} U(x) \widehat{P} \sqrt{U(x)}$ & $(\mathrm{BBQT})$ & {$[11]$} \\
\hline
\end{tabular}


Using the identity (2.11) and the representations (3.2) and (3.3), one can easily find that

$$
\begin{aligned}
{\left[\mathcal{Q}_{\alpha}, \mathcal{Q}_{\alpha}^{\dagger}\right] } & =U(x) \mathcal{W}_{\alpha}^{\prime}(x)+\frac{1-2 \alpha}{2} U(x) U^{\prime \prime}(x), \\
{\left[\mathcal{H}_{\alpha}, \mathcal{Q}_{\alpha}\right] } & =-\left[\mathcal{Q}_{\alpha}, \mathcal{Q}_{\alpha}^{\dagger}\right] \mathcal{Q}_{\alpha}, \\
{\left[\mathcal{H}_{\alpha}, \mathcal{Q}_{\alpha}^{\dagger}\right] } & =\mathcal{Q}_{\alpha}^{\dagger}\left[\mathcal{Q}_{\alpha}, \mathcal{Q}_{\alpha}^{\dagger}\right]
\end{aligned}
$$

which require that the algebra of three operators $\mathcal{Q}_{\alpha}, \mathcal{Q}_{\alpha}^{\dagger}$ and $\mathcal{H}_{\alpha}$ is nothing but the generally deformed oscillator algebra (GDOA) [29].

Although our approach holds for any $\alpha$ of (3.8), we shall focus our attention on the effect changing the SUSY parameter $\alpha$ to $1-\alpha$ via the identification: $\alpha \mapsto \bar{\alpha}=1-\alpha$, with $0 \leq \bar{\alpha} \leq 1$. Then (3.6) become

$$
\begin{aligned}
& \mathcal{Q}_{\alpha} \rightarrow \overline{\mathcal{Q}}_{\alpha} \equiv \mathcal{Q}_{1-\alpha}=\frac{1}{\sqrt{2}}\left(U^{1-\alpha}(x) \frac{d}{d x} U^{\alpha}(x)+\mathcal{W}_{1-\alpha}(x)\right), \\
& \mathcal{Q}_{\alpha}^{\dagger} \rightarrow \overline{\mathcal{Q}}_{\alpha}^{\dagger} \equiv \mathcal{Q}_{1-\alpha}^{\dagger}=\frac{1}{\sqrt{2}}\left(-U^{\alpha}(x) \frac{d}{d x} U^{1-\alpha}(x)+\mathcal{W}_{1-\alpha}(x)\right),
\end{aligned}
$$

where the corresponding effective potential

$$
\begin{aligned}
\overline{\mathcal{V}}_{\alpha}(x)-\epsilon & =\frac{1}{2} \mathcal{W}_{1-\alpha}^{2}(x)-\frac{1}{2} U(x) \mathcal{W}_{1-\alpha}^{\prime}(x)-\frac{1-2 \alpha}{2} U^{\prime}(x) \mathcal{W}_{1-\alpha}(x), \\
\overline{\mathcal{V}}_{\mathrm{U}}^{(\alpha)}(x) & =\frac{\alpha(\alpha-1)}{2} U^{\prime 2}(x)-\frac{\alpha}{2} U^{\prime \prime}(x) U(x),
\end{aligned}
$$

and the associated GDOA is the same as (3.9) except that they differ in the sign of the term $1-2 \alpha$.

There is another side to this identification. Indeed a simple inspection leads us to make a crucial observation that a such changing in the SUSY parameter $\alpha$ is nothing but an accidental symmetry under the $\mathbb{Z}_{2}$ action, if and only if $\alpha=1 / 2$ is the unique fixed point of this transformation. For example, the emergence of this kind of striking symmetry has already been discussed by Fiset and Hussin [37]. They find that this symmetry has an interesting and particular effects on the coherent states built out of SUSY potential functions. Here by an accidental symmetry, we mean that this symmetry in not predicted neither by factorization method, nor the SUSY approach. As we can see, selecting an appropriate SUSY parameter $\alpha$ can also be interpreted as a consequence of the striking and accidental symmetry under the $\mathbb{Z}_{2}$ action, where only $\bar{\alpha}=0,1 / 2$, and 1 belong to the set (3.8). It is obvious that the most suitable ordering parameter $\alpha$ is given by the simplest case $\alpha=1 / 2$, (i.e., $a=b=-1 / 4$, see Table 1 ), and corresponding to the well-known kinetic operator, 
$\widehat{T}_{1 / 2}=\frac{1}{2}\left(\sqrt{U(x)} \frac{d}{d x} \sqrt{U(x)}\right)^{2}$, which has deserved special attention in research papers over the years (see, for example [11] and references therein.)

To be more precise about this transformation, let us introduce an operator $\widehat{\mathcal{Z}}$ that implements the $\mathbb{Z}_{2}$-transformation on the annihilation operator $\mathcal{Q}_{\alpha}$. Thus, acting on $\mathcal{Q}_{\alpha}$, we must require that the implement operator $\widehat{\mathcal{Z}}$ satisfies

$$
\widehat{\mathcal{Z}} \mathcal{Q}_{\alpha} \widehat{\mathcal{Z}}^{-1}=\mathcal{Q}_{1-\alpha} \quad \Longrightarrow \quad \widehat{\mathcal{Z}} \mathcal{Q}_{\alpha}=\mathcal{Q}_{1-\alpha} \widehat{\mathcal{Z}}
$$

with $\widehat{\mathcal{Z}}^{-1} \neq \widehat{\mathcal{Z}}^{\dagger}$, since we assume that they are no other operators, apart from $\mathcal{Q}_{\alpha}$, which are affected by the $\mathbb{Z}_{2}$ action. Note that we have also demand the invariance for the fixed point $\alpha=1 / 2$, i.e., the commutator $\left[\widehat{\mathcal{Z}}, \mathcal{Q}_{1 / 2}\right]=0$, which means that the operators $\widehat{\mathcal{Z}}$ and $\mathcal{Q}_{1 / 2}$ share the same eigenfunctions. Then, along with (3.13), it is obvious that the implement operator $\widehat{\mathcal{Z}}$ is identified to $\mathcal{Q}_{1 / 2}$ for $\alpha=1 / 2$. However it acts as an intertwiner transformation for the ladder operators $\mathcal{Q}_{\alpha}$ and $\mathcal{Q}_{1-\alpha}$ for $\alpha \neq 1 / 2$. Moreover, acting (3.13) on the right-hand side by a function $\phi_{n}(x)$, we get

$$
\Psi_{n}(x) \equiv \mathcal{Q}_{1-\alpha} \psi_{n}(x)=\widehat{\mathcal{Z}}\left(\mathcal{Q}_{\alpha} \varphi_{n}(x)\right) \equiv \widehat{\mathcal{Z}} \Phi_{n}(x)
$$

where $\psi_{n}(x) \equiv \widehat{\mathcal{Z}} \varphi_{n}(x)$. Clearly, we look for $\widehat{\mathcal{Z}}$ as an intertwiner operator. For the sake of completeness, we now seek the intertwining operator in the form of a first-order lineardifferential operator

$$
\widehat{\mathcal{Z}}=\frac{1}{\sqrt{2}}\left(F(x) \frac{d}{d x} F(x)+G(x)\right)
$$

where $F(x)$ and $G(x)$ are two real functions to be determined, such that $\widehat{\mathcal{Z}}$ fulfills (3.13). For lack of space, the implement operator is evaluated and the result is

$$
\widehat{\mathcal{Z}} \equiv \mathcal{T}_{\alpha}(x) \widehat{\nabla}_{x}^{(\alpha)} \mathcal{T}_{\alpha}(x)
$$

with

$$
\begin{array}{ll}
\mathcal{T}_{\alpha}(x) & =U^{1-\alpha}(x) \exp \left\{\int^{\mu(x)} \frac{\mathcal{W}_{\alpha}(\eta)-\mathcal{W}_{1-\alpha}(\eta)}{2} d \mu(\eta)\right\}, \\
\widehat{\nabla}_{x}^{(\alpha)} & =\frac{1}{\sqrt{2}}\left(\frac{d}{d x}+\frac{1}{2} \frac{\mathcal{W}_{\alpha}(x)+\mathcal{W}_{1-\alpha}(x)}{U(x)}\right) .
\end{array}
$$

It is worth noting that if $\alpha=1 / 2$, then (3.17a $)$ is reduced to $\mathcal{T}_{1 / 2}(x)=U^{1 / 2}(x) \equiv$ $m^{-1 / 4}(x)$ and the implement operator $\widehat{\mathcal{Z}}$ becomes the standard annihilation operator $\mathcal{Q}_{1 / 2}$, 
(see Table 1), i.e.,

$$
\widehat{\mathcal{Z}}=\mathcal{Q}_{1 / 2}=\frac{1}{\sqrt{2}}\left(\sqrt{U(x)} \frac{d}{d x} \sqrt{U(x)}+\mathcal{W}_{1 / 2}(x)\right) .
$$

\section{PDM CS AND GENERALIZED POSITION-MOMENTUM UNCERTAINTY RELATION}

Now the main question is: what kind of position-dependent mass coherent states (PDM CS) are expected in both symmetries? This section is devoted to construct a set of PDM CS for isospectral Hamiltonians $\mathcal{H}_{\alpha}$. As mentioned in the introduction, there are three equivalent definitions of CS and one of the candidates (Klauder-Perelomov's approach) looks on CS as an orbit of the ground-state $|0\rangle$, under the Weyl-Heisenberg displacement operator $\mathcal{D}(z)$ expressed in terms of ladder operators.

We have seen that $\mathcal{Q}_{\alpha}$ and $\mathcal{Q}_{\alpha}^{\dagger}$ are such operators and can be used to derive their associated PDM CS. Then the related PDM CS, $|z ; \alpha\rangle$, for which we are looking for must verify the eigenvalue equation $\mathcal{Q}_{\alpha}|z ; \alpha\rangle=z|z ; \alpha\rangle$ and annihilates the ground-state $\left|\Xi_{0} ; \alpha\right\rangle$, i.e., $\mathcal{Q}_{\alpha}\left|\Xi_{0} ; \alpha\right\rangle=0$.

Using (3.6) the ground-state $\left|\Xi_{0} ; \alpha\right\rangle$ can be calculated straightforwardly by integration, and we get

$$
\left|\Xi_{0} ; \alpha\right\rangle \sim U^{\alpha-1}(x) \exp \left\{-\int^{\mu(x)} \mathcal{W}_{\alpha}(\eta) d \mu(\eta)\right\},
$$

up to normalization constant. In Klauder-Perelomov's approach, our PDM CS are expressed through

$$
|z ; \alpha\rangle=\mathcal{D}_{\alpha}(z)\left|\Xi_{0} ; \alpha\right\rangle
$$

and one assumes that the displacement operator, $\mathcal{D}_{\alpha}(z)$, takes the form [29]

$$
\mathcal{D}_{\alpha}(z)=\exp \left\{i \mathcal{S}_{\alpha}(z)\right\}, \quad \text { with } \quad \mathcal{S}_{\alpha}(z)=-i z \mathcal{K}_{\alpha},
$$

where $\mathcal{K}_{\alpha}$ is unknown operator which can be determined using the unitarity condition of $\mathcal{D}_{\alpha}(z)$, i.e., $\mathcal{D}_{\alpha}^{-1}(z)=\mathcal{D}_{\alpha}^{\dagger}(z)$. Indeed this restriction leads us to identity both $\mathcal{S}_{\alpha}(z)$ and $\mathcal{K}_{\alpha}$ as hermitian operators if and only if $z=-z^{*}$, i.e., $\Re(z)=0$. Having introduced the form of $\mathcal{D}_{\alpha}(z)$, we are now able to state the following proposition [29] in order to determine $\mathcal{K}_{\alpha}$. 
Proposition 1 Let $z \in i \mathbb{R}$. Then, for every displacement operator $\mathcal{D}_{\alpha}(z)$ as defined in (4.3) and acting on the ladder operators under the general scheme:

$$
\mathcal{D}_{\alpha}^{\dagger}(z) \mathcal{Q}_{\alpha} \mathcal{D}_{\alpha}(z)=\mathcal{Q}_{\alpha}+z\left[\mathcal{Q}_{\alpha}, \mathcal{Q}_{\alpha}^{\dagger}\right], \quad \text { and } \quad \mathcal{D}_{\alpha}^{\dagger}(z) \mathcal{Q}_{\alpha}^{\dagger} \mathcal{D}_{\alpha}(z)=\mathcal{Q}_{\alpha}^{\dagger}+z^{*}\left[\mathcal{Q}_{\alpha}, \mathcal{Q}_{\alpha}^{\dagger}\right]
$$

must verify

$$
\left[\mathcal{S}_{\alpha}(z), \mathcal{Q}_{\alpha}\right]=i z\left[\mathcal{Q}_{\alpha}, \mathcal{Q}_{\alpha}^{\dagger}\right], \quad \text { and } \quad\left[\mathcal{S}_{\alpha}(z), \mathcal{Q}_{\alpha}^{\dagger}\right]=i z^{*}\left[\mathcal{Q}_{\alpha}, \mathcal{Q}_{\alpha}^{\dagger}\right]
$$

where $z=-z^{*}$.

Proof. Let us define the operator $\mathcal{P}_{\alpha}=\left[\mathcal{Q}_{\alpha}, \mathcal{D}_{\alpha}(z)\right]$. Expanding $\exp \left\{i \mathcal{S}_{\alpha}(z)\right\}$ in the development of Taylor series, we find

$$
\mathcal{P}_{\alpha}=-\sum_{k=0}^{+\infty} \frac{i^{k}}{k !}\left[\mathcal{S}_{\alpha}^{k}(z), \mathcal{Q}_{\alpha}\right]
$$

and using (4.5), a straightforward calculation yields to the recursion relation satisfying

$$
\left[\mathcal{S}_{\alpha}^{k}(z), \mathcal{Q}_{\alpha}\right]=i k \mathcal{S}_{\alpha}^{k-1}(z)\left[\mathcal{Q}_{\alpha}, \mathcal{Q}_{\alpha}^{\dagger}\right]
$$

and by inserting (4.7) into (4.6), we have

$$
\mathcal{P}_{\alpha}=z \mathcal{D}_{\alpha}(z)\left[\mathcal{Q}_{\alpha}, \mathcal{Q}_{\alpha}^{\dagger}\right]
$$

On the other hand, starting from $\mathcal{P}_{\alpha}=\left[\mathcal{Q}_{\alpha}, \mathcal{D}_{\alpha}(z)\right]$ and multiplying both sides on the left by $\mathcal{D}_{\alpha}^{\dagger}(z)$ and comparing with (4.4), we get 4.8). This completes the proof.

The substitution of $\mathcal{S}_{\alpha}(z)=-i z \mathcal{K}_{\alpha}$ into (4.5) yields four possible and distinguishable solutions for $\mathcal{K}_{\alpha}: \mathcal{Q}_{\alpha}, \mathcal{Q}_{\alpha}^{\dagger}$ and $\mathcal{Q}_{\alpha} \pm \mathcal{Q}_{\alpha}^{\dagger}$. The two-first cases will be omitted to avoid illdefined hermiticity condition imposed to $\mathcal{K}_{\alpha}$, while the last case with a positive sign is that in which we are interested in. Under these conditions, the displacement operator (4.3) can be expressed as:

$$
\mathcal{D}_{\alpha}(z)=\exp \left\{z\left(\mathcal{Q}_{\alpha}+\mathcal{Q}_{\alpha}^{\dagger}\right)\right\}_{z=-z^{*}}=\exp \left\{z \mathcal{Q}_{\alpha}-z^{*} \mathcal{Q}_{\alpha}^{\dagger}\right\}
$$

and by inserting (4.1) and (4.9) into PDM CS (4.2), we get

$$
|z ; \alpha\rangle \sim U^{\alpha-1}(x) \exp \left\{\sqrt{2} z \mathcal{W}_{\alpha}(x)+\frac{1-2 \alpha}{\sqrt{2}} z U^{\prime}(x)-\int^{\mu(x)} \mathcal{W}_{\alpha}(\eta) d \mu(\eta)\right\} .
$$


This expression is the general form of isospectral Hamiltonians CS endowed with PDM for an arbitrary quantum system and already deduced in the case $\alpha=1 / 2$ in [29]. Let us now turn to the construction of PDM CS for an accidental symmetry, $\overline{|z ; \alpha\rangle}$, under the $\mathbb{Z}_{2}$ action and satisfying the eigenvalue equation $\mathcal{Q}_{1-\alpha} \overline{z ; \alpha\rangle}=z \mid \overline{z ; \alpha\rangle}$. This procedure can easily be achieved in the same manner it was performed in the exact SUSY symmetry or merely by performing the substitution $\alpha \mapsto \bar{\alpha}=1-\alpha$ in (4.10). Finally, we get the relationship between both coherent states

$$
\begin{aligned}
\overline{|z ; \alpha\rangle} & \sim U^{-\alpha}(x) \exp \left\{\sqrt{2} z \mathcal{W}_{1-\alpha}(x)-\frac{1-2 \alpha}{\sqrt{2}} z U^{\prime}(x)-\int^{\mu(x)} \mathcal{W}_{1-\alpha}(\eta) d \mu(\eta)\right\} \\
& =U^{1-2 \alpha}(x) \exp \left\{-\sqrt{2} z \widetilde{\mathcal{W}}_{\alpha}(x)-\sqrt{2}(1-2 \alpha) z U^{\prime}(x)+\int^{\mu(x)} \widetilde{\mathcal{W}}_{\alpha}(\eta) d \mu(\eta)\right\}|z ; \alpha\rangle,
\end{aligned}
$$

where $\widetilde{\mathcal{W}}_{\alpha}(x)=\mathcal{W}_{\alpha}(x)-\mathcal{W}_{1-\alpha}(x)$

On the other hand, the relevance of (3.13) is clear in the sense that if $|z ; \alpha\rangle$ is PDM CS of $\mathcal{Q}_{\alpha}$, then $\widehat{\mathcal{Z}}|z ; \alpha\rangle$ is a new PDM CS of $\mathcal{Q}_{1-\alpha}$ under the $\mathbb{Z}_{2}$-transformation. Indeed by acting (3.13) on $|z ; \alpha\rangle$, taking into account that $\mathcal{Q}_{\alpha}|z ; \alpha\rangle=z|z ; \alpha\rangle$, it seems that $\overline{|z ; \alpha\rangle}=$ $\widehat{\mathcal{Z}}|z ; \alpha\rangle$ solves the new eigenvalue PDM CS equation, where the action of $\widehat{\mathcal{Z}}$ on $|z ; \alpha\rangle$ is well established in (4.11). However by comparing (4.10) to (4.11), it is easy to verify that $\overline{|z ; 1-\alpha\rangle}=|z ; \alpha\rangle$, so that $\overline{|z ; \alpha\rangle}=\widehat{\mathcal{Z}}|z ; 1-\alpha\rangle$. The same identity can be deduced for the state $|z ; \alpha\rangle$, i.e., $|z ; \alpha\rangle=\widehat{\mathcal{Z}}^{-1}|z ; 1-\alpha\rangle$.

As we can see, the $\mathbb{Z}_{2}$ action acts on PDM CS (4.10) as a functional factor representing the implementation of the transformation and affects (4.10) as long as $\alpha \neq 1 / 2$ and $U(x)$ remains a function. Otherwise, (i.e., $\alpha=1 / 2$ ), the $\mathbb{Z}_{2}$ action is broken and both PDM CS in (4.11) are reduced to be the same state.

In the following one may prove that PDM CS $|z ; \alpha\rangle$ of (4.10) minimize the generalized position-momentum uncertainty relation (GUR). To prove this, let us calculate the variances, $\left(\Delta \mathcal{W}_{\alpha}\right)^{2}$ and $\left(\Delta \Pi_{\alpha}\right)^{2}$, of the superpotential and generalized momentum operators given by

$$
\widehat{\mathcal{W}}_{\alpha}(x)=\frac{1}{\sqrt{2}}\left(\mathcal{Q}_{\alpha}+\mathcal{Q}_{\alpha}^{\dagger}\right)-\frac{1-2 \alpha}{\sqrt{2}} U^{\prime}(x), \quad \text { and } \quad \widehat{\Pi}_{\alpha}(x)=-\frac{i}{\sqrt{2}}\left(\mathcal{Q}_{\alpha}-\mathcal{Q}_{\alpha}^{\dagger}\right),
$$

and deduced from (3.6). By definition, the variance of an operator, say $\widehat{\Theta}$, is defined as: $(\Delta \Theta)^{2}=\left\langle z ; \alpha\left|\widehat{\Theta}^{2}\right| z ; \alpha\right\rangle-\langle z ; \alpha|\widehat{\Theta}| z ; \alpha\rangle^{2}$. Then with the help of the first equation of (3.9), 
we find after some straightforward but lengthy calculation

$$
\begin{aligned}
\left\langle\mathcal{W}_{\alpha}\right\rangle & \equiv\left\langle z ; \alpha\left|\widehat{\mathcal{W}}_{\alpha}\right| z ; \alpha\right\rangle=-\frac{1-2 \alpha}{2} U^{\prime}(x), \\
\left\langle\Pi_{\alpha}\right\rangle & \equiv\left\langle z ; \alpha\left|\widehat{\Pi}_{\alpha}\right| z ; \alpha\right\rangle=-i \sqrt{2} z \\
\left\langle\mathcal{W}_{\alpha}^{2}\right\rangle & \equiv\left\langle z ; \alpha\left|\widehat{\mathcal{W}}_{\alpha}^{2}\right| z ; \alpha\right\rangle=-\frac{1}{2} U(x) \mathcal{W}_{\alpha}^{\prime}(x)-(1-2 \alpha) U^{\prime}(x) \mathcal{W}_{\alpha}(x) \\
& +\frac{1-2 \alpha}{2} U(x) U^{\prime \prime}(x)-\frac{(1-2 \alpha)^{2}}{4} U^{\prime 2}(x), \\
\left\langle\Pi_{\alpha}^{2}\right\rangle & \equiv\left\langle z ; \alpha\left|\widehat{\Pi}_{\alpha}^{2}\right| z ; \alpha\right\rangle=-2 z^{2}+\frac{1}{2} U(x) \mathcal{W}_{\alpha}^{\prime}(x)+\frac{1-2 \alpha}{4} U(x) U^{\prime \prime}(x),
\end{aligned}
$$

keeping in mind that $z=-z^{*}$. Thus the use of definition of the variance given above provides the product

$$
\left(\Delta \mathcal{W}_{\alpha}\right)^{2} \cdot\left(\Delta \Pi_{\alpha}\right)^{2}=\frac{1}{4}\left(\left\langle z ; \alpha\left|\left[\mathcal{Q}_{\alpha}, \mathcal{Q}_{\alpha}^{\dagger}\right]\right| z ; \alpha\right\rangle\right)^{2}-\frac{1}{2} \mathcal{R}_{\alpha}(x)\left\langle z ; \alpha\left|\left[\mathcal{Q}_{\alpha}, \mathcal{Q}_{\alpha}^{\dagger}\right]\right| z ; \alpha\right\rangle,
$$

where the function $\mathcal{R}_{\alpha}(x)$ is defined as

$$
\mathcal{R}_{\alpha}(x)=(1-2 \alpha) U^{\prime}(x)\left(\mathcal{W}_{\alpha}(x)+\frac{1-2 \alpha}{2} U^{\prime}(x)\right) .
$$

As we can see a deeper insights are necessary on the function $\mathcal{R}_{\alpha}(x)$, if we are interested to saturate and minimize (4.14).

Saturation of (4.14). Using (4.15), it is worth noting that PDM CS $|z ; \alpha\rangle$ saturate GUR if and only if $\mathcal{R}_{\alpha}(x)=0$. This latter leads us to consider three requirements: (R1) $\alpha=1 / 2,(\mathbf{R} 2) U^{\prime}(x)=0$, and/or $(\mathbf{R} 3) \mathcal{W}_{\alpha}(x)=-(1-2 \alpha) U^{\prime}(x) / 2$. It is easy to convince ourselves that (R2) must be avoided since in this case the quantum system loses its PDM features. However if the restriction $(\mathbf{R} 1)$ is satisfied, then $(\mathbf{R} 3)$ yields $\mathcal{W}_{1 / 2}(x)=0$ which corresponds to a free particle in (3.7a). This is in contrast with our study since the case $\alpha=1 / 2$ has its own PDM potential and can not be zero. Thus in order to avoid this ambiguity, we suggest that $\mathcal{W}_{\alpha}(x) \neq-(1-2 \alpha) U^{\prime}(x) / 2$ simultaneously with $\alpha=1 / 2$. On the other hand, taking into account only the restriction (R3) we get from (3.7)

$$
\mathcal{V}_{\text {eff }}(x)=-\frac{1}{4} U(x) U^{\prime \prime}(x)-\frac{1}{8} U^{\prime 2}(x)+\epsilon,
$$

which is independent of $\alpha$. We conclude that the saturation of PDM CS (4.10) is possible if and only if:

- $\alpha=1 / 2$ and $\mathcal{W}_{\alpha}(x) \neq-(1-2 \alpha) U^{\prime}(x) / 2$,

- $\alpha \neq 1 / 2$ and $\mathcal{W}_{\alpha}(x)=-(1-2 \alpha) U^{\prime}(x) / 2$, leading to the effective potential (4.16). 
Minimization of (4.14). On the other context, it is also simple to verify that PDM $\mathrm{CS},|z ; \alpha\rangle$, minimize GUR as follows

$$
\left(\Delta \mathcal{W}_{\alpha}\right)^{2} \cdot\left(\Delta \Pi_{\alpha}\right)^{2} \geq \frac{1}{4}\left(\left\langle z ; \alpha\left|\left[\mathcal{Q}_{\alpha}, \mathcal{Q}_{\alpha}^{\dagger}\right]\right| z ; \alpha\right\rangle\right)^{2}
$$

if and only if $\mathcal{R}_{\alpha}(x)<0$. This restriction leads us to distinguish between two possible cases from (4.15) as follows (with $0 \leq \alpha \leq 1$ ):

(C1) If $(1-2 \alpha) U^{\prime}(x)<0$, then $\mathcal{W}_{\alpha}(x)+(1-2 \alpha) U^{\prime}(x) / 2>0$,

(C2) If $(1-2 \alpha) U^{\prime}(x)>0$, then $\mathcal{W}_{\alpha}(x)+(1-2 \alpha) U^{\prime}(x) / 2<0$.

The first condition of (C1) gives either $-1 \leq 1-2 \alpha<0$ with $U^{\prime}(x)>0$, or $0<1-2 \alpha \leq 1$ for $U^{\prime}(x)<0$. Then, in both situations, the second condition yields $\mathcal{W}_{\alpha}(x)>0$. The same analysis is made for (C2), which gives either $-1 \leq 1-2 \alpha<0$ with $U^{\prime}(x)<0$, or $0<1-2 \alpha \leq 1$ for $U^{\prime}(x)>0$, while the second condition of this case yields $\mathcal{W}_{\alpha}(x)<0$. We conclude here that the sign of the superpotential $\mathcal{W}_{\alpha}(x)$ is entirely controlled by the minimization of GUR obtained from 4.10).

\section{CONCLUDING REMARKS}

We have extended the ideas given in [33] for an arbitrary quantum system of isospectral family of Hamiltonians endowed with PDM and constructed their PDM CS through the unitary transformation.

In this paper, our main aim is to emphasize the greatest importance of introducing the unitary transformation and its consequence for solving the ordering problem. For instances, we have shown that the profile of the mass function $m(x)$ (resp. $U(x)$ ) is subjected to singularities (resp. zeros). In this way, we have been able to define a special set of the SUSY parameter $\alpha$ which distinguishes different choices of the kinetic operator $\widehat{T}_{\alpha}$ and some of the mostly used forms are reported in table 1 . We have also observed the occurrence of an accidental symmetry under the $\mathbb{Z}_{2}$-transformation if the special value $\alpha=1 / 2$ is the unique fixed point of this transformation and proved that this point is the most suitable choice under this set. This last comment may be the most subtle idea in our paper because it suggests that the $\mathbb{Z}_{2}$ action is rather simple for explaining the origin of the very special

ordering $\widehat{T}_{1 / 2}=\frac{1}{2} \sqrt{U(x)} \widehat{P} U(x) \widehat{P} \sqrt{U(x)}$, due to the special feature of the point $\alpha=1 / 2$. 
We have seen that there is another remarkable property concerning the fixed point, that is

the operator $\widehat{\mathcal{Z}}$ which implements the $\mathbb{Z}_{2}$ action acts as $\mathcal{Q}_{1 / 2}$ if $\alpha=1 / 2$, however it plays the role of an intertwiner operator between $\mathcal{Q}_{\alpha}$ and $\mathcal{Q}_{1-\alpha}$ for each $\alpha \neq 1 / 2$ of the set $\mathfrak{S}_{\alpha_{n}}$.

We have constructed PDM CS in the Klauder-Perelomov's sense through the exact SUSY symmetry and proved that the $\mathbb{Z}_{2}$ action acts on them as a functional factor as long as $\alpha \neq 1 / 2$. Finally we have also shown that the fixed point has another remarkable effect; its associated PDM CS saturate the generalized position-momentum uncertainty relation, while this latter is minimized for $\alpha \neq 1 / 2$. A quite important result of this minimization is that the sign of the superpotential $\mathcal{W}_{\alpha}(x)$ is entirely determined.

1 B. Mielnik, J. Math. Phys. 25, 3387 (1984).

2 M. M. Nieto, Phys. Lett. 145B, 208 (1984).

3 S. H. Dong, Factorization Method in Quantum Mechanics (The Netherlands, Springer, 2007) and references therein.

4 K. Chadan and P. C. Sabatier, Inverse Problems in Quantum Scattering Theory (Springer, Berlin, 1977).

5 F. Cooper, A. Khare and U. Sukhatme, Phys. Rep. 251, 267 (1995).

6 F. Iachello, Lie Algebra and Applications. Lect. Notes Phys. 708 (Springer, Berlin, 2006).

7 R. Bravo and M. S. Plyushchay, Phys. Rev. D 93, 105023 (2016) and references therein.

8 J.-M. Lévy-Lebland, Phys. Rev. A 52, 1845 (1995); Eur. J. Phys. 13, 215 (1992).

9 B. Roy and P. Roy, J. Phys. A: Math. Gen. 35, 3961 (2002).

10 R. Koç and M. Koka, J. Phys. A: Math. Gen. 36, 8105 (2003).

11 B. Bagchi, B. Banerjee, C. Quesne and V. M. Tkachuk, J. Phys. A: Math. Gen. 38, 2929 (2005).

12 A. Gunguly and L. M. Nieto, J. Phys. A: Math. Theor. 40, 7265 (2007).

13 A. A. Suzko and A. Schulze-Halberg, Phys. Lett. A 372, 5865 (2008).

14 S.-A. Yahiaoui and M. Bentaiba, Int. J. Theor. Phys. 48, 315 (2009).

15 B. Midya and B. Roy, Phys. Lett. A 373, 4117 (2009).

16 G. Lévai and O. Özer, J. Math. Phys. 51, 092103 (2010).

17 S. H. Mazharimousavi, Phys. Rev. A 85, 034102 (2012).

18 P. Ring and P. Schuck, The Nuclear Many Body Problems (Springer, New York, 1980), p. 211. 
19 C. Quesne and V. M. Tkachuk, J. Phys. A: Math. Gen. 37, 4267 (2004).

20 L. Jiang, L.-Z. Yi and C.-S. Jia, Phys. Lett. A 345, 279 (2005).

21 O. Mustafa and S. H. Mazharimousavi, J. Phys. A: Math. Theor. 41, 244020 (2008) and references therein.

22 P. Harrison, Quantum Wells, Wires and Dots. Theoretical and Computational Physics of Semiconductor Nanostructures (John Wiley \& Sons, LTD, 2005).

23 G. Bastard, Wave Mechanics Applied to Semiconductor Heterostructures (Les Ulis: Éditions de physique, 1998).

24 O. von Roos, Phys. Rev. B 27, 7547 (1983).

25 E. Schrödinger, Naturwiss 14, 664 (1926).

26 R. J. Glauber, Phys. Rev. 131, 2766 (1963).

27 D. J. Fernández, V. Hussin and L. M. Nieto, J. Phys. A: Math. Gen. 27, 3547 (1994).

28 J.-P. Gazeau, Coherent States in quantum Mechanics (Wiley-VCH, Weinheim, 2009).

29 S.-A. Yahiaoui and M. Bentaiba, J. Phys. A: Math. Theor. 47, 025301 (2014); J. Phys. A: Math. Theor. 45, 444034 (2012).

30 V. C. Ruby and M. Senthilvelan, J. Math. Phys. 51, 52106 (2010).

31 S. Cruz y Cruz and O. Rosas-Ortiz, J. Phys. A: Math. Theor. 42, 185205 (2009); Int. J. Theor. Phys. 50, 2201 (2011).

32 N. Amir and S. Iqbal, J. Math. Phys. 56, 062108 (2015); Commun. Theor. Phys. 66, 41 (2016).

33 M. S. Kumar and A. Khare, Phys. Lett. A 217, 73 (1996).

34 R. A. Morrow and K. R. Brownstein, Phys. Rev. B 30, 678 (1984).

35 Q. J. Zhu and H. Kroemer, Phys. Rev. B 27, 3519 (1983).

36 D. J. BenDaniel and C. B. Duke, Phys. Rev. B 152, 683 (1966).

37 M.-A. Fiset and V. Hussin, J. Phys.: Conf. Ser. 624, 012016 (2015) 